\title{
Desenvolvimento de aplicações m-Learning nas plataformas J2ME e Flash Lite.
}

\author{
Leandro Ramos de Oliveira ${ }^{12}$ \\ Roseclea Duarte Medina ${ }^{1}$ \\ ${ }^{1}$ Curso de Ciência da Computação - Centro de Tecnologia - Universidade Federal de \\ Santa Maria (UFSM) \\ ${ }^{2}$ Bolsista do Programa de Educação Tutorial (PET) \\ leandroo@inf.ufsm.br, rose@inf.ufsm.br
}

\section{Resumo}

Este artigo apresenta uma alternativa de implementação de um objeto de aprendizagem que é utilizado em ambiente desktop, para que possa também ser visualizado e utilizado por usuários de dispositivos móveis, colocando em prática o conceito de m-Learning. Para este trabalho, foi utilizada a plataforma de desenvolvimento para dispositivos móveis J2ME, e a plataforma Flash Lite, bem como simuladores de telefones celulares presentes em ambas.

Palavras chaves: $\mathrm{m}$-learning, $\mathrm{j} 2 \mathrm{me}$, flash lite, objetos de aprendizagem, dispositivos móveis.

\begin{abstract}
This article presents an alternative implementation of an learning object that is used in desktop environment, so that it can also be viewed and used by users of mobile devices, putting into practice the concept of m-learning. For this work, the development platforms for mobile devices used where J2ME and Flash Lite, as well as cell phones simulators present in both.
\end{abstract}

Keywords: m-learning, j2me, flash lite, learning objects, mobile devices.

\section{Introdução}

Não é recente o fato de informática e educação andarem lado a lado em prol de uma disseminação cada vez maior de informação, cultura e saber, de forma fácil e rápida. Porém agora, com o advento da computação móvel, um novo conceito para esta união vem à tona, o chamado m-Learning. M-Learning, do inglês mobile learning, é a utilização de dispositivos móveis tais como telefones celulares, Personal Digital Assistants (PDAs) e smatphones, no processo de ensino e aprendizagem. Este novo paradigma pode ser aplicado não apenas em ambientes de estudo tais como escolas e 
universidades, mas também em âmbito empresarial, por exemplo, dando suporte a capacitação e qualificação de funcionários.

Durante os últimos anos, enquanto as pesquisas sobre m-Learning estavam apenas iniciando, educadores utilizavam-se plenamente da informática para $o$ desenvolvimento dos chamados objetos de aprendizagem. Ou seja, aplicações utilizadas para divulgação de conteúdos educacionais. Tais objetos são plenamente visualizados em computadores pessoais, porém a maioria perde grande parte de seus recursos, e alguns chegam a tornarem-se totalmente inúteis quando acessados através de dispositivos móveis. Por isso, profissionais da área da computação vêm buscando desenvolver alternativas de implementação de tais objetos, que possibilitam que uma grande quantidade de objetos educacionais já desenvolvidos possam ser visualizados com a totalidade de seus recursos também em dispositivos móveis, permitindo desta forma, uma aplicação do conceito de m-Learning.

Neste artigo, pretende-se apresentar alternativas de implementação de um objeto de aprendizagem, já utilizado em ambiente desktop, que permita o mesmo além de ser visualizado em um dispositivo móvel, oferecer possibilidades de interação para o usuário, proporcionando um processo de ensino e aprendizagem mais dinâmico e completo. Para tal finalidade, foram utilizadas as plataformas de desenvolvimento para dispositivos móveis J2ME, pertencente à empresa Sun Microsystems, e Flash Lite, pertencente à empresa Adobe Systems Incorporated. Além disso, devido ao fato de poucas implementações com estas intenções terem sido desenvolvidas, a validação do objeto desenvolvido também apresenta uma comparação crítica entre o objeto apresentado no desktop e o do dispositivo móvel, ensejando com este trabalho, produzir uma análise sobre a implementação e utilização deste objeto, destacando seus pontos críticos de forma que possa contribuir para a produção de trabalhos futuros.

Devido o conceito de m-Learning ainda ser recente, pode-se considerar que a produção científica relacionada ainda está em sua fase inicial. Com isso, a maioria dos trabalhos até agora já desenvolvidos, aborda a parte teórica deste novo paradigma, e poucos trazem implementação de aplicações que se enquadram no mesmo. Este trabalho preocupa-se em apresentar e impulsionar o desenvolvimento de aplicações que colocam em prática a questão de produção de conteúdos educacionais para dispositivos móveis.

No restante deste artigo, é apresentada inicialmente uma revisão conceitual sobre m-Learning, destacando questões de padronização, exemplos de aplicações e alguns projetos relacionados. Pretende-se ainda expor uma análise sobre as plataformas de desenvolvimento para dispositivos móveis J2ME, destacando sua arquitetura e questões de implementação, e Flash Lite. Além disso, será destacada uma breve análise de um objeto de aprendizagem para desktop, bem como alternativas de implementação do mesmo nas duas plataformas aqui abordadas. Por fim, será apresentada uma análise geral dos resultados obtidos, além de conclusões e trabalhos futuros.

\section{M-Learning}

A educação sempre foi uma das principais preocupações e prioridades para uma sociedade que busca enriquecimento em todos os aspectos. O "envelhecimento do conhecimento" e a crescente necessidade de capacitação, formação e atualização profissional, aliada à exigência de mobilidade, contribuiu para o surgimento do mLearning. A conceituação de m-Learning pode ser facilmente entendida pela união dos 
conceitos de aprendizagem e dispositivos móveis. Assim, m-Learning é todo aprendizado que é sustentado através de dispositivos portáteis, que possuem sua própria fonte de energia, e que podem ser facilmente utilizados onde não haja acesso a conexões de rede física (Driscoll e Carliner, 2005). Isso não implica na exigência de utilização de redes wireless, pois o dispositivo pode oferecer o conteúdo educacional armazenado em seu hardware, possibilitando o aprendizado móvel, porém off-line.

Um dos principais problemas relativos à evolução de trabalhos que se utilizam deste novo paradigma, é o fato de ainda não se ter uma formulação concreta sobre padronização dos meios de acesso a informação. Devido ao grande número de aparelhos móveis disponíveis no mercado com acesso a internet, a produção de conteúdo para tais dispositivos torna-se bastante limitada por variáveis como: fabricante do aparelho, sistema operacional que utiliza e no caso de telefones e smartphones, a operadora prestadora do serviço telefônico. Várias organizações internacionais como, Learning Technologies Standardization Committee (LTSC) da IEEE, European Committee for Standardization (ECS) e a subcomissão 36 do primeiro encontro da International Standardization Organization, em associação ao International Electrotechnical Commission Committee (ISO/IEC JTC1 SC36), são exemplos de esforços para elaboração de padrões para o desenvolvimento de conteúdos a serem visualizados em dispositivos móveis em geral. Porém, os resultados mais comuns são a utilização de URLs que podem ser adaptadas possibilitando uma boa visualização em qualquer dispositivo, tanto móvel quanto fixo. Exemplo disso são os trabalhos desenvolvidos pelo grupo Mobile Web Initiative (MWI) pertencente a W3C, que buscam a definição de uma web única, baseada em regras, que quando seguidas por autores e desenvolvedores permitem que o conteúdo produzido seja visualizado em qualquer dispositivo (W3C, 1994).

Apesar da questão da falta de padronização, investir para o sucesso de mLearning é válido e necessário. Pelo fato de lidar com recursos como internet, dispositivos móveis e educação, já difundidos e aceitos pela grande maioria das pessoas, o ambiente disponível para aplicação deste conceito é bastante vasto. Podemos destacar como exemplo do emprego de m-Learning a sua aplicação em projetos e grupos de trabalhos colaborativos; como uma forma diferente de ensino em escolas e universidades em relação a livros e computadores; na educação à distância; em campanhas de conscientização; como suporte a capacitação de funcionários em empresas; dentre outras (Tribal, 1998). Pode-se ainda destacar projetos que já estão sendo desenvolvidos pelo mundo, tais como a produção de aplicações móveis pela City University of Hong Kong (CityU), com foco em tornar a aprendizagem dos estudantes de Hong Kong mais flexível, aproveitando a grande utilização da tecnologia pelos mesmos (Vogel, Kennedy, Kuan, Kwok, Lai, 2007). E ainda, um projeto desenvolvido pela empresa Ericsson, que busca avaliar a aceitação deste novo conceito pelo mercado através de cursos sobre m-Learning (Ericsson, 2005).

Como visto nesta seção, a questão de m-Learning, apesar de ainda sofrer com algumas dificuldades com padrões, é um campo que ainda tem muito a ser explorado, devido a grande quantidade de áreas de aplicação. Na próxima seção, será levantada uma alternativa aos desenvolvedores para que possam produzir conteúdos a serem utilizados nessas áreas. Serão destacadas questões como recursos oferecidos, arquitetura da plataforma, e avaliação da mesma no quesito de produção de conteúdos educacionais para aparelhos móveis.

\section{Plataforma J2ME}


Java 2 Micro Edition (J2ME) é uma plataforma inteiramente projetada com o intuito de atender as necessidades de desenvolvedores da área de computação móvel. Pertencente a empresa Sun Microsystems, trata-se de uma versão reduzida da plataforma padrão Java 2 Standard Edition (J2SE), que permite a produção de aplicações para dispositivos móveis tais como telefones celulares, PDAs, smartphones, dentre outros. O que estes dispositivos têm em comum é o fato de possuírem pouco poder de processamento e pouca memória para suportar a J2SE usada em computadores desktop e servidores, daí a necessidade de uma versão reduzida para a produção de materiais para tais dispositivos. Além disso, compreende interfaces de usuário, modelos de segurança, protocolos de comunicação em rede e outras funcionalidades que, quando combinadas, constituem um ambiente de execução Java otimizado para uso de memória, processamento e operações de entrada e saída (Bevilacqua, 2007).

\subsection{Arquitetura}

Um dos desafios enfrentados pela equipe da Sun, Java Community Process (JCP), no momento em que estavam desenvolvendo a plataforma J2ME, foi o de encontrar uma maneira para que uma mesma aplicação desenvolvida em J2ME pudesse ser visualizada em qualquer um dos tantos dispositivos móveis presentes no mercado. Pelo fato destes dispositivos não seguirem uma padronização de hardware, como a seguida por computadores desktop, cada fabricante desenvolve o aparelho a seu gosto, o que dificulta muito o desenvolvimento de aplicações genéricas. partes:

A solução encontrada pela JCP foi a de separar a arquitetura da J2ME em duas

- Configuração (Configurations): que trata o ambiente de execução (Java RunTime Environment) e possui um pacote de classes que trabalha sobre cada dispositivo, chamado core. Ainda, é subdividida em dois blocos, um que opera sobre dispositivos com pouco poder computacional, CLDC (Connected Limited Device Configuration), e outro que opera sobre dispositivos com maior poder computacional, CDC (Connected Device Configuration);

- Perfil (Profile): que consiste em um pacote de classes que permitem aos desenvolvedores encontrar as características de um grupo de dispositivos móveis, e adaptar suas aplicações a eles.

\subsection{Desenvolvimento de objetos de aprendizagem}

Uma das principais vantagens da plataforma J2ME é possibilitar que toda aplicação desenvolvida seja acessível de qualquer dispositivo móvel. Este questão garante que um objeto de aprendizagem móvel possa ser utilizado independente do aparelho do usuário. Por exemplo, se um telefone celular é compatível com Java, ele converte automaticamente o código Java e o executa, sem ter de interpretá-lo e adaptalo a arquitetura do aparelho o que restringiria o seu funcionamento apenas a certos telefones. 
Além disso, a J2ME, apesar de ser uma versão simplificada da J2SE, apresenta uma boa quantidade de recursos que auxiliam o desenvolvedor de objetos. Dentre estes podemos destacar a facilidade que o programador tem de estruturar os elementos na tela conforme sua vontade, definindo suas posições absolutas, a facilidade de criação de imagens fornecida pelo uso da classe Graphics e a facilidade de entendimento das Interfaces de Programação de Aplicativos (APIs), o que permite o desenvolvimento rápido de aplicações.

Todos estes recursos são de grande valia para o desenvolvimento de objetos de aprendizagem, e foram explorados no desenvolvimento das atividades deste trabalho. Na próxima seção é descrita uma analise da plataforma Flash Lite.

\section{Plataforma Flash Lite}

Flash Lite é uma tecnologia toda projetada para dispositivos móveis, onde busca aliar um bom desempenho de processamento com os poucos recursos apresentados por tais dispositivos. Atualmente está disponível em duas versões: Flash Lite 1.0 e Flash Lite 1.1 baseados no Flash Player 4, e Flash Lite 2 baseado no Flash Player 7. Segundo documento divulgado na Internet pela Adobe, dentre os vários recursos disponibilizados no Flash Lite 2, destaca-se o suporte a conexões via sockets, manipulação de arquivos XML, suporte a Actionscript 2.0, formatação de texto melhorada e suporte a Unicode. Além disso possui recursos de multimídia (imagem, áudio e vídeo) e armazenamento de dados para reuso (Adobe Systems Incorporated, 2007). O Flash Lite facilita o trabalho de profissionais que já desenvolviam conteúdos em Flash para ambientes desktop, pois podem se valer do conhecimento adquirido, relativo ao desenvolvimento na plataforma Flash para realizarem trabalhos para dispositivos móveis.

Como qualquer plataforma emergente, o Flash Lite ainda apresenta alguns pontos negativos a serem considerados, como: indisponibilidade, que se refere ao fato de poucos celulares apresentarem suporte a conteúdos Flash Lite, principalmente no Brasil; inconsistência de versões: dependendo do aparelho, marca e sistema operacional, o Flash Lite não poderá ser visualizado plenamente em todos seus modos; política de distribuição: o fato de usuários que compram aparelhos que saem de fábrica sem suporte ao Flash Lite, terem de pagar dez dólares para obtê-lo, dificulta a aceitação da tecnologia; e limitações do plug-in: o Actionscript presente no Flash Lite oferece uma gama menor de recursos comparado a sua versão desktop.

Apesar das limitações, o Flash Lite permite a criação de interfaces ricas e muito bem elaboradas em poucos minutos, além de não exigir um grande conhecimento de programação para tal finalidade, fato esse que também contribui para a utilização do mesmo no processo de desenvolvimento de objetos de aprendizagem para dispositivos móveis.

\section{Atividades desenvolvidas}

O presente trabalho enseja apresentar uma adaptação de um objeto que é utilizado em ambiente desktop e foi projetado para tal, possibilitando que este também possa ser acessado através de dispositivos móveis. Para a realização deste trabalho, foi 
escolhido um dentre os vários objetos educacionais presentes no repositório de objetos da Rede Interativa Virtual de Educação (RIVED), que é um dos mais conhecidos programas de desenvolvimento de conteúdos pedagógicos digitais do Brasil.

Nas próximas seções, será apresentada uma análise geral do objeto de aprendizagem escolhido, bem como uma alternativa de re-implementação do mesmo para dispositivos móveis nas plataformas J2ME e Flash Lite, e ainda uma análise final dos resultados obtidos.

\subsection{Objeto de aprendizagem "Roda Viva"}

Para este trabalho, foi escolhido o objeto de aprendizagem "Roda Viva", disponível no site do RIVED (http://rived.mec.gov.br). Este objeto tem como público alvo alunos da primeira série do Ensino Médio e objetiva introduzir questões relacionadas aos movimentos físicos dos corpos (força, mecânica e movimento), para que possam ser discutidas e debatidas em sala de aula. Além disso, por se tratar de uma atividade inicial, o objeto em questão é relativamente simples, tratando-se apenas de uma transição de imagens e questões relativas ao tema. Por isso, foram utilizados recursos das linguagens de marcação HTML e XML, e uma animação em Flash para a transição de imagens (RIVED, 2007). Segue abaixo uma figura de como este objeto é visualizado em ambiente desktop a uma resolução de tela de 1152 por 864 pixels.

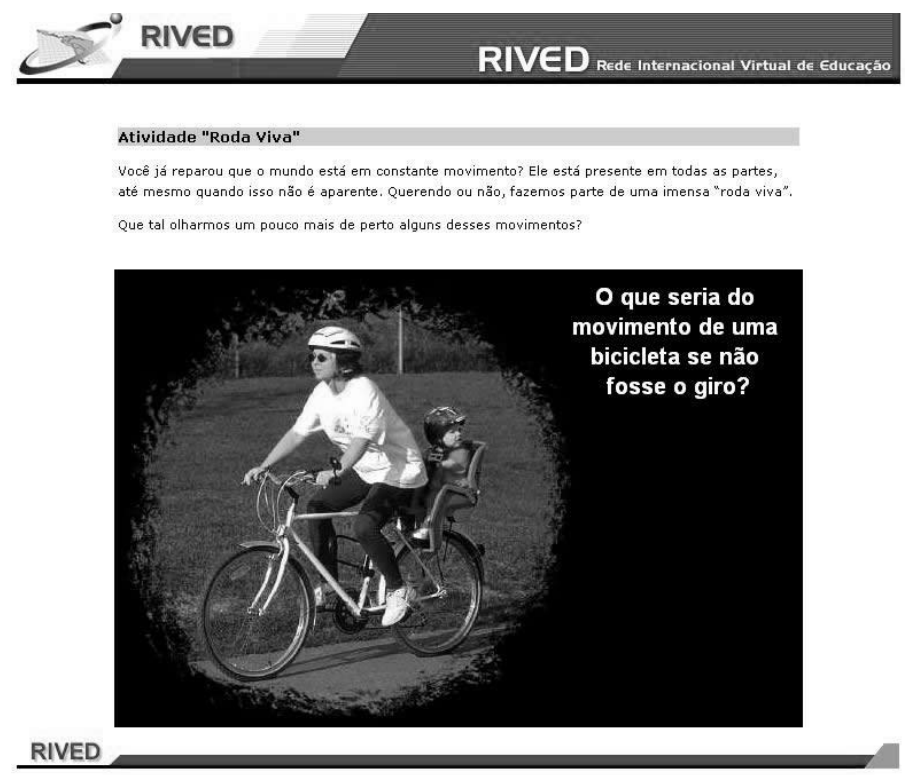

Figura 1 - Visualização de objeto em ambiente desktop.

Como este objeto foi totalmente planejado para o ambiente desktop, podemos observar na Figura 1, que o mesmo apresenta boas características de visualização. Desta forma, o objeto mantém a totalidade de seus recursos, permitindo aos alunos que o utilizarem uma boa interação. 


\subsection{Implementação do objeto em J2ME}

Uma alternativa para visualizarmos o objeto "Roda Viva" em um dispositivo móvel, seria implementa-lo levando-se em conta desde o início este novo ambiente. Para tal propósito, foi utilizada a plataforma de desenvolvimento para dispositivos móveis J2ME, bem como o pacote de ferramentas Wireless Toolkit 2.5 for CLDC, ambos da Sun Microsystems.

Com base na análise anterior do objeto de aprendizagem, projetou-se a reimplementação do mesmo, tentando manter ao máximo suas características, adaptando somente o necessário para uma boa visualização e interação por parte do usuário. Por isso, como o objeto trata-se basicamente de uma transição de imagens e textos contínua, desenvolveu-se este mesmo recurso, porém agora, deixando a cargo do usuário o momento de passar para a próxima imagem e texto, pressionando a seta da direita no teclado direcional do aparelho, ou retroceder para a imagem e texto anterior, pressionando a seta da esquerda. Além disso, manteve-se o título, o cabeçalho da atividade e o rodapé sempre visível, alterando somente as imagens e seus textos correspondentes. Essa alternativa permite que se utilize apenas uma tela para todo o objeto, diminuindo desta forma o tamanho do mesmo.

Segue abaixo, uma imagem do objeto "Roda Viva" adaptado para dispositivos móveis usando J2ME, sendo visualizado no simulador presente no Wireless Toolkit 2.5 for CLDC.

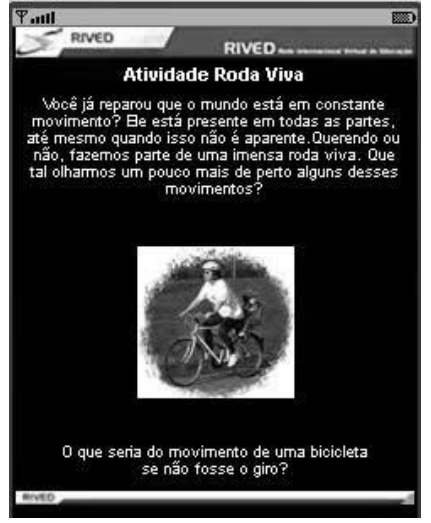

Figura 2 - Alternativa de implementação do objeto de aprendizagem "Roda Viva" em J2ME.

Analisando a Figura 2, percebemos que quando o objeto é projetado para ser visualizado em um ambiente móvel, pode-se trabalhar a melhor forma de superar dificuldades como tamanho da tela e o recurso de implementação a ser utilizado. A implementação do objeto "Roda Viva" em J2ME é relativamente simples, pois não utiliza grandes recursos de animações, interatividade e imagens complexas, o que possibilita manter toda sua funcionalidade sem tornar o objeto pesado para ser acessado via rede wireless.

\subsection{Implementação do objeto em Flash Lite}


Agora, em contrapartida a alternativa de visualização do objeto "Roda Viva" em J2ME, é apresentado uma opção de implementação do mesmo em Flash Lite. Os testes sobre a implementação foram realizados no próprio simulador de telefones celulares presente no Flash Professional 8. Veja a Figura 3 abaixo.
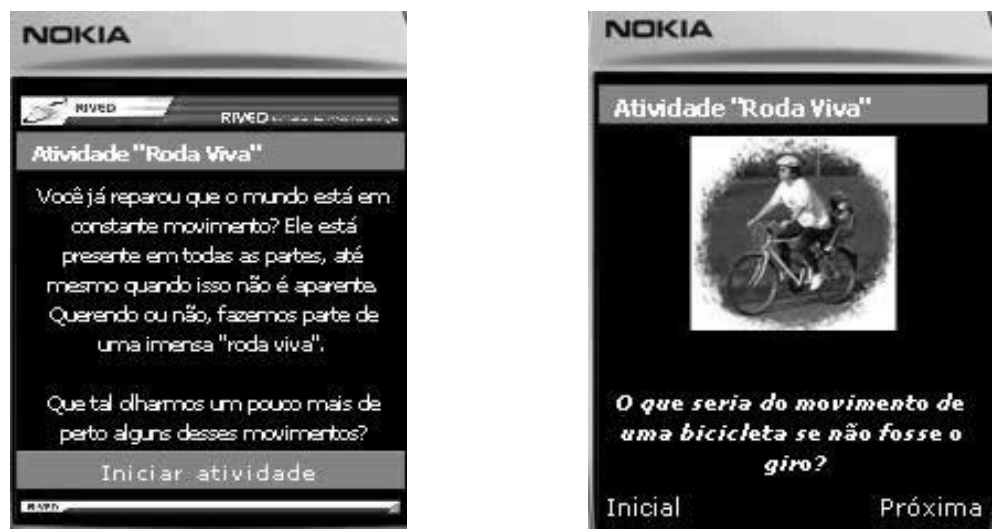

Figura 3 - Alternativa de implementação do objeto de aprendizagem "Roda Viva" em Flash Lite (tela inicial a esquerda e tela da atividade a direita).

Neste caso, foi escolhido um dos modelos Nokia suportados pelo simulador presente no Flash, que é o Nokia 7610. Além disso, foi ativada via código à opção de o objeto ser apresentado em tela cheia.

Analisando o resultado obtido, percebe-se que o conteúdo do objeto em questão, apesar de não possuir uma grande área de trabalho, se acomoda de forma harmônica na pequena tela, e possibilita uma plena interação com o usuário. Nota-se ainda que mesmo em dispositivos diferentes, a visualização do conteúdo é a mesma, apesar de estarmos trabalhando com dispositivos de uma mesma empresa. Outro ponto a destacar é o uso de animação na transição das imagens. Ou seja, quando o usuário pressiona a tecla para ir para a próxima imagem, a atual desliza para cima até sair da tela, seguida logo abaixo pela próxima que desliza para cima até chegar ao centro da tela.

Para esta implementação, utilizou-se uma tela inicial de apresentação do objeto e com um botão para iniciar a atividade. E uma segunda tela que seria a atividade propriamente dita, com a transição das imagens e seus respectivos textos.

\subsection{Análise geral}

Traçando agora um paralelo entre as duas implementações apresentadas neste artigo, podemos destacar alguns pontos que são relevantes quando se trata de desenvolvimento para dispositivos móveis.

Como foram abordadas formas diferentes de projeto para o objeto de aprendizagem "Roda Viva" nas duas plataformas, a questão de boa definição das imagens torna-se difícil de observar. Isto porque na implementação com J2ME todo o objeto se resume a uma única tela, o que reduz o espaço disponível para acomodação das imagens, implicando em serem de tamanho menor, em contrapartida à implementação em Flash Lite que se utiliza de duas telas. 
Outra questão que podemos observar é o uso de animações. Tanto a plataforma J2ME quanto a plataforma Flash Lite possuem suporte a criação de animações. Porém, como o desenvolvimento em Flash Lite ocorre através do programa Flash, a criação de animações acaba por se tornar mais fácil nesta plataforma, visto que o Flash sempre teve, através de sua linha de tempo, um bom suporte para este tipo de criação. Já em J2ME, as animações são todas criadas via código, ou seja, sem uma interface mais amigável para o desenvolvedor.

Pode-se ainda ressaltar a questão referente à realização dos testes das aplicações produzidas. Ambas as plataformas trazem consigo simuladores que permitem ao desenvolvedor testar seus trabalhos em simulações de dispositivos móveis reais. Esta questão é bastante importante, pois evita que para se testar uma aplicação seja necessário dispor de um dispositivo real e ainda transferir toda vez o conteúdo desenvolvido para o mesmo.

Por fim, o mais importante a destacar se refere à compatibilidade dos conteúdos desenvolvidos em cada plataforma com os dispositivos móveis presentes no mercado. Como já mencionado nas seções específicas sobre cada plataforma, pode-se destacar uma pequena vantagem da $\mathrm{J} 2 \mathrm{ME}$ sobre o Flash Lite, no que se refere à compatibilidade. A J2ME, por ter surgido a mais tempo que o Flash Lite, já abrange um número maior de dispositivos que suportam aplicações desenvolvidas nela. Este fato acaba por induzir o desenvolvimento deste tipo de conteúdo para a plataforma J2ME. Porém, com o grande avanço apresentado pelo Flash Lite nos últimos anos, a tendência é que esta nova tecnologia seja cada vez mais aceita pelo mercado, o que reduziria esta diferença.

\section{Considerações finais}

Com base no que foi levantado neste artigo, pode-se perceber que o conceito de m-Learning e sua aplicação estão cada vez mais difundidos. O desenvolvimento de objetos de aprendizagem próprios para dispositivos móveis é de grande valia tanto para educadores, que passam a dispor de mais um ambiente para promover a educação, quanto para profissionais da computação, que são estimulados a desenvolver trabalhos que possam contribuir para o desenvolvimento desta nova tecnologia. Além disso, percebe-se que esta parceria entre educação e informática pode facilitar a aceitação dos dispositivos móveis pela sociedade, promovendo uma maior concentração dos trabalhos relativos à padronização de tais dispositivos, facilitando desta forma todo e qualquer desenvolvimento de aplicações móveis, que não apenas objetos educacionais, visto que se baseiam em uma mesma plataforma.

Este trabalho preocupou-se em apresentar o estado atual relativo a m-Learning, ressaltando questões de implementação de objetos de aprendizagem. Como o objeto desenvolvido foi testado apenas em simuladores, no futuro pretende-se melhorar a implementação do objeto de forma a superar as dificuldades presentes, e então testa-lo em um dispositivo móvel, possibilitando assim, ter uma idéia concreta da totalidade de recursos realmente disponíveis bem como dificuldades encontradas na sua implementação.

\section{Rferências bibliográficas}


Driscoll, M.; Carliner, S. Advanced Web-Based Training Strategies. 2005.

Georgiev, T.; Georgieva, E.; Trajkovski, G. Transitioning from e-Learning to mLearning: Present Issues and Future Challenges. 2006.

Hadzilacos, T.; Tryfona, N. Constructive m-Learning Environments. 2005.

Holzinger, A.; Nischelwitzer, A.; Meisenberger, M. Mobile Phones as a Challenge for m-Learning: Examples for Mobile Interactive Learning Objects (MILOs). 2005.

Knudsen, J.; Li, S. Beginning J2ME: From Novice to Professional, $3^{\text {th }}$ edition. 2005.

Keogh, J. J2ME: The Complete Reference.

W3C Mobile Web Initiative. Disponível em: <http://www.w3.org/Mobile/>. Acesso em: out. 2007.

Tribal How can I use M-learning?. Disponível em: <http://www.mlearning.org/how/how-can-i-use-m-learning-.htm>. Acesso em: out. 2007.

Vogel, D.; Kennedy, D.; Kuan, K.; Kwok, R.; Lai, J. Do Mobile Device Applications Affect Learning. 2007.

Ericsson Mobile Learning: The Next Generation Of Learning. Disponível em: < http://learning.ericsson.net/mlearning2/> Acesso em: out. 2007.

Legget, R.; Boer, D., W.; Janousek, S. Foundation Flash Applications for Mobile Devices. New York: 2006.

Mikkonen, T. Programming Mobile Devices An Introduction For Practitioners. Chichester: 2007.

Bevilacqua, F. Jogo de Estratégia Multi-jogador para Telefones Celulares, no Estado do Rio Grande do Sul. Santa Maria: UFSM, 2007. 57p. Trabalho de Graduação.

RIVED. Rede Interativa Virtual de Educação. Apresenta textos sobre objetos de aprendizagem. Disponível em: <http://rived.proinfo.mec.gov.br/>. Acesso em: out. 2007. 
RIVED. Rede Interativa Virtual de Educação. Objeto de aprendizagem "Roda Viva". Disponível em:

http://rived.proinfo.mec.gov.br/atividades/fisica/conservacao/atividade1/atividade1.htm >. Acesso em: out. 2007.

ADOBE SYSTEMS INCORPORATED. Adobe Flash Lite. Disponível em: <http://www.adobe.com/products/flashlite/>. Acesso em: out. 2007.

ADOBE SYSTEMS INCORPORATED. Adobe Flash Lite 2.1 Datasheet. Disponível em: $\quad$ <http://www.adobe.com/products/flashlite/productinfo/overview/datasheet.pdf $>$. Acesso em: out. 2007. 